\section{Percepção da imagem corporal de crianças e adolescentes com diferentes níveis socio- econômicos na cidade de Florianópolis, Santa Catarina, Brasil}

\author{
Body image perception in children and \\ adolescents with different socio-economic \\ status in the city of Florianópolis, in the \\ State of Santa Catarina, Brazil
}

Érico Felden Pereira 1

Susane Graup 2

Adair da Silva Lopes 3

Adriano Ferreti Borgatto 4

Luciane Sanchotene Etchepare Daronco 5
1 Laboratório de Cronobiologia Humana. Departamento de
Fisiologia. Universidade Federal do Paraná. Centro Politécnico.
Jardins das Américas. Curitiba, PR, Brasil. CEP: 81.531-990.
E-mail: ericofelden@yahoo.com.br
2 Programa de Pós-Graduaça em Engenharia de Produção.
Universidade Federal de Santa Catarina. Florianópolis, SC, Brasil.
3,4 Programa de Pós-Graduação em Educação Física. Universidade
Federal de Santa Catarina. Florianópolis, SC, Brasil.
5 Núcleo de Estudos em Medidas e Avaliação da Educação Física.
Universidade Federal de Santa Maria. Santa Maria, RS, Brasil.

\section{Abstract}

Objectives: to identify the body image perception in children and adolescents from different socioeconomic backgrounds.

Methods: a cross-cutting study was carried out in the city of Florianópolis, in the State of Santa Catarina, Brazil, in the year 2006. Four hundred and two schoolchildren, with an average age of $11.35 \pm$ 1.98 years, enrolled in the final years of primary school were investigated. Body image perception was identified using the Stunkard silhouettes protocol and socio-economic status was evaluated using the criteria proposed by the National Pres and Research Association (ANEP). Prevalence ratios were calculated for dissatisfaction arising from perceived excessive weight or excessive thinness.

Results: among males, silhouette 3 was the one most often chosen among those from the upper and middle classes, whereas those of lower socioeconomic status opted for silhouette 4; middle and upper class girls, however, opted for silhouette 2 was chosen and girls from less privileged backgrounds tended to select silhouette 3 as best representing their own silhouette. Silhouette 3 for males and 2 for females are considered to be the ideal for most school-age children. Less privileged young people had a higher prevalence of dissatisfaction arising from excess weight than more privileged ones.

Conclusions: there was a tendency to children of a higher social status to be dissatisfied because they regarded themselves as being too thin and for children of lower social status to be dissatisfied because they perceived themselves to be overweight.

Key words Body image, Socioeconomic factors, Perception, Child, Adolescent

\section{Resumo}

Objetivos: identificar a percepção da imagem corporal em diferentes níveis socioeconômicos de crianças e adolescentes.

Métodos: estudo transversal realizado no municipio de Florianópolis, Santa Catarina, no ano de 2006. Quatrocentos e dois escolares, com média de idade de 11,35 \pm 1,98 anos, matriculados nas séries finais do ensino fundamental, foram investigados. A percepção da imagem corporal foi identificada por meio do protocolo de silhuetas de Stunkard e o nivel socioeconômico pelos critérios propostos pela Associação Nacional de Empresas e Pesquisa (ANEP). Foram calculadas razões de prevalências para insatisfação pelo excesso de peso e pela magreza.

Resultados: no sexo masculino, a silhueta 3 foi a mais citada nas classes alta e média e a 4 na baixa; no feminino a silhueta 2 foi a mais citada nas classes alta e baixa e a 3 na média considerando a percepção real de sua silhueta. A silhueta 3 para o sexo masculino e a 2 para o feminino foram consideradas ideais pelo maior percentual de escolares. Os jovens da classe baixa apresentaram maiores prevalências de insatisfação pelo excesso de peso em relação os da classe alta.

Conclusões: houve uma tendência dos escolares das classes mais altas apresentarem insatisfação pela magreza e das mais baixas pelo excesso de peso.

Palavras-chave Imagem corporal, Fatores socioeconômicos, Percepção, Criança, Adolescente 


\section{Introdução}

O conceito de corpo saudável ou bonito vem sofrendo transformações ao longo da história e os padrões de beleza de outrora são bastante diferenciados dos atuais. No entanto, o culto ao corpo, de uma forma ou de outra, sempre esteve associado à imagem de poder, beleza e mobilidade social. $\mathrm{Na}$ atualidade, é possível identificar uma crescente insatisfação das pessoas com a própria aparência, num contexto no qual há uma grande valorização de corpos fortes e magros.

A imagem corporal pode ser considerada a forma que o corpo se apresenta para cada sujeito e sua determinação é formada, tanto em relação a si próprio, como em relação às outras pessoas. 1 Pode ser influenciada por inúmeros fatores de origem física, psicológica, ambiental e cultural dentro da subjetividade de cada sujeito, tais como sexo, idade, meios de comunicação, crenças, raça e valores. ${ }^{2}$

Evidências científicas apontam que a insatisfação com a imagem corporal é cada vez mais comum, principalmente para o sexo feminino. ${ }^{3} \mathrm{Em}$ especial na adolescência, as mudanças físicas, psicológicas e sociais podem afetar significantemente os hábitos alimentares, a saúde nutricional e percepção do próprio corpo. O rápido crescimento exige ao mesmo tempo um aporte nutricional diferenciado e uma adaptação social e cultural nas atividades diárias. A mídia em geral desempenha um papel importante na vida dos adolescentes e dissemina a idéia de uma perfeição corporal, na qual a magreza simboliza competência, sucesso e atração sexual, enquanto a obesidade representa a preguiça, autopiedade e menor poder de decisão e qualidade de vida. 4

A insatisfação com a imagem corporal é apontada como um fator de risco para depressão e baixa autoestima nas diferentes faixas da adolescência. ${ }^{4,5}$ Estudos com populações européias, norte-americanas, orientais e brasileiras têm identificado prevalências de insatisfação com imagem corporal em crianças e adolescentes em torno de $20 \%$ a $60 \%$ dependendo de fatores como o sexo, etnia, estado nutricional e socioeconômico e também às diferentes formas de avaliação. ${ }^{3,4,6-12}$

O nível socioeconômico é apontado como importante variável no estudo da insatisfação com a imagem corporal em crianças e adolescentes. ${ }^{12}$ Apesar disso, embora exista uma tendência cada vez mais forte de maior insatisfação nas classes mais baixas, principalmente em relação ao excesso de peso, essa relação parece sofrer alterações de acordo com a etnia investigada. ${ }^{7}$ No Brasil, um aumento de sobrepeso e obesidade nas classes sociais mais baixas foi identificado. ${ }^{13}$ No entanto, análises de percepção da imagem corporal em diferentes classes socioeconômicas são escassos.

Diante disso, considerando a carência de estudos que investiguem a relação entre percepção da imagem corporal e o nível socioeconômico, bem como a importância de se conhecer essa relação para o trabalho com crianças e adolescentes, buscou-se nesta pesquisa identificar a percepção da imagem corporal em diferentes classes socioeconômicas em crianças e adolescentes do município de Florianópolis.

\section{Métodos}

Trata-se de um estudo transversal realizado com crianças e adolescentes matriculados em escolas públicas e privadas de Florianópolis, Santa Catarina, Brasil. Esse município é atualmente a capital brasileira com o melhor Índice de Desenvolvimento Humano (0,875). Possui, aproximadamente, $350 \mathrm{mil}$ habitantes e estima-se que em torno de $97 \%$ da população de crianças e adolescentes estejam frequentando a educação básica regular. 14

A amostra do estudo foi formada por 402 crianças e adolescentes; 183 do sexo masculino e 219 do sexo feminino, matriculados nas séries finais do ensino fundamental, na faixa etária de nove a 15 anos. A presente pesquisa faz parte de um macroprojeto desenvolvido no período de 2004 a 2006 com crianças e adolescentes de escolas públicas e privadas de Florianópolis, intitulado "Indicadores de Crescimento Físico e Aptidão Física Relacionada à Saúde de Escolares de Florianópolis". Os protocolos de intervenção foram aprovados pelo Comitê de Ética em Pesquisa da Universidade Federal de Santa Catarina e acompanham normas da Resolução 196/96 do Conselho Nacional de Saúde para pesquisas com seres humanos.

Para definição da amostra para o macroprojeto foram realizados os seguintes procedimentos: estratificação por região (centro, continente e interior); característica da escola (pública e particular) e série de ensino ( $5^{\mathrm{a}}$ a $8^{\mathrm{a}}$ série). Para determinação do tamanho amostral, recorreu-se ao modelo proposto por Luiz e Magnanini 15 para investigações epidemiológicas, e considerou-se a prevalência de excesso de peso de $20 \%$, nível de confiança de 1,96 (Intervalo de Confiança 95\%). A precisão adotada em torno da prevalência estimada foi de $3 \%$ e um acréscimo de $20 \%$ como possível índice de perda. Como a amostragem foi realizada por conglomerado, definiu-se um efeito do desenho igual a 1,5 e encontrou-se o 
número mínimo necessário de 1214. As análises de percepção da imagem corporal foram adicionadas no último ano de coleta de dados (entre abril e outubro de 2006) e realizadas em aproximadamente $1 / 3$ da amostra total do projeto e em 11 escolas (quatro estaduais, quatro municipais e três particulares). As turmas em cada escola sorteada foram selecionadas de forma aleatória.

As escolas sorteadas foram informadas sobre os objetivos do estudo e os procedimentos que seriam realizados. Após autorização da direção, os alunos foram informados sobre o propósito da pesquisa e receberam o termo de consentimento livre e esclarecido para ser assinado e preenchido por seus responsáveis e devolvido no dia seguinte. Todas as crianças e adolescentes matriculadas nas escolas sorteadas foram elegíveis ao estudo; no entanto, foram excluídos os escolares que faltaram à aula no dia da coleta, que não entregaram o termo de consentimento livre e esclarecido assinado pelos pais ou responsáveis e/ou questionário socioeconômico, que se recusaram em participar da pesquisa e os escolares que não informaram o sexo, idade ou preencheram erroneamente a escala de silhuetas. Além disso, em função da necessidade de realização de testes de aptidão física para o macroprojeto, os jovens incapacitados de realizarem as testagens e as gestantes também foram excluídos da amostra. Dessa forma, o número de sujeitos para cada idade foi o seguinte: 9 anos $(n=66) ; 10$ anos $(n=82) ; 11$ anos $(n=87) ; 12$ anos $(\mathrm{n}=80) ; 13$ anos $(\mathrm{n}=58) ; 14$ anos $(\mathrm{n}=20) ; 15$ anos $(\mathrm{n}=9)$.

A equipe de coleta de dados recebeu treinamento para a coleta de todas as variáveis e foi formada por pesquisadores vinculados ao Núcleo de Pesquisa em Atividade Física e Saúde da Universidade Federal de Santa Catarina.

A percepção da imagem corporal foi analisada pela escala de nove silhuetas, proposta por Stunkard et al.,16 a qual representa um continuum desde a magreza (silhueta 1) até a obesidade severa (silhueta 9), conforme apresentado na Figura 1.

\section{Figura 1}

Conjunto de silhuetas proposto para avaliação da imagem corporal.
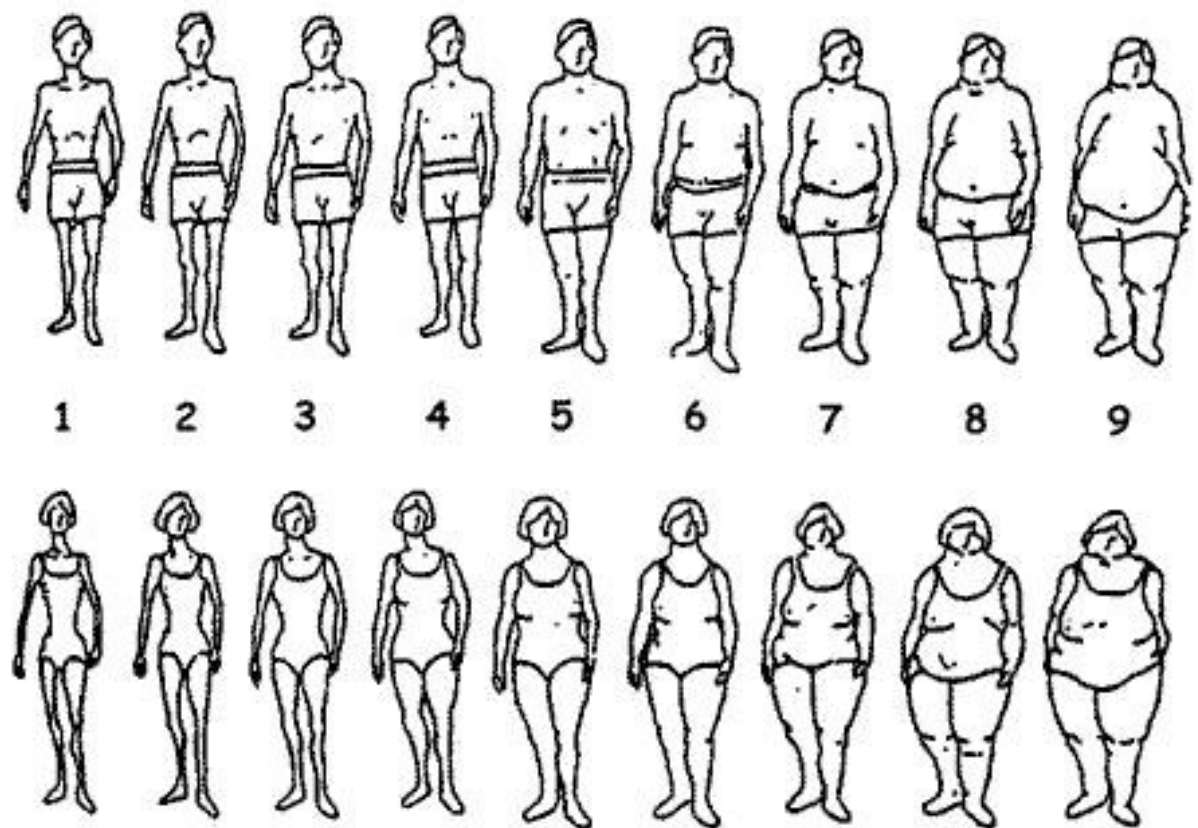

6

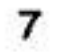

8

9

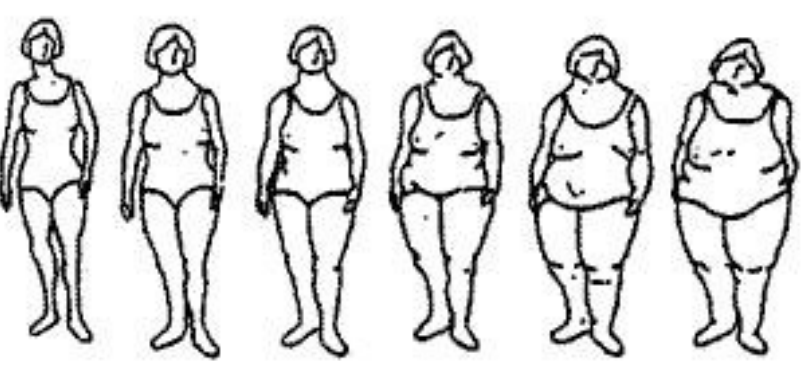

Fonte: Stunkard AJ. et al. Use of Danish adoption register for the study of obesity and thinness. In: Key SS et al. The genetics of neurological. New York; 1983.16 
Nessa escala, o indivíduo escolhe o número da silhueta que considera semelhante a sua aparência corporal real (Percepção da Imagem Corporal Real PICR) e também o número da silhueta que acredita ser mais condizente a sua aparência corporal ideal (Percepção da Imagem Corporal Ideal - PICI). Para a avaliação da satisfação corporal subtraiu-se da aparência corporal real a aparência corporal ideal, podendo esse número variar de -8 até +8 . Caso essa variação fosse igual a zero, o indivíduo era classificado como satisfeito com sua aparência e se diferente de zero classificava-se como insatisfeito. Caso a diferença fosse positiva considerava-se uma insatisfação pelo excesso de peso e, quando negativa, uma insatisfação pela magreza.

O cálculo de nível socioeconômico seguiu as recomendações propostas pela Associação Nacional de Empresas e Pesquisa (ANEP) ${ }^{17}$ por meio dos Critérios de Classificação Econômica Brasil. Essa análise considera a posse de bens móveis e o grau de escolaridade do chefe da família, classificando nas classes A1, A2, B1, B2, C, D e E. Para esse estudo as classes foram agrupadas considerando classe alta (A1, A2, B1 e B2), média (C) e baixa (D e E).

$\mathrm{O}$ real estado nutricional foi avaliado com o intuito de fornecer uma melhor caracterização da amostra. Como medidas antropométricas foram realizadas coletas da massa corporal e estatura. A massa corporal foi medida utilizando-se uma balança digital da marca Plenna Glass, com escalas de resolução de $100 \mathrm{~g}$. A estatura foi aferida utilizando-se um estadiômetro com procedimento padronizado. A definição de sobrepeso e obesidade seguiu as recomendações propostas por Cole et al.,18 que utiliza os valores do Índice de Massa Corporal (IMC) analisados de acordo com a idade e o sexo. Optou-se por essa classificação pois os autores, para elaborá-la, utilizaram dados de diferentes países, entre eles o Brasil, Grã-Bretanha, Holanda e Estados Unidos, além de disponibilizar valores de corte para crianças e adolescentes. Como a análise do real estado nutricional não é foco principal de nossa avaliação, foi utilizada uma classificação em duas categorias (peso normal e sobrepeso/obesidade).

Os dados das percepções reais e ideais e do nível de satisfação com a imagem corporal, tanto considerando o grupo geral como separados por sexo, foram analisados quanto a sua distribuição, utilizando-se da estatística W de Shapiro-Wilk, que revelou distribuição normal $(p>0,05)$. Visando identificar valores de tendência central (média) e de dispersão (desvio padrão) foi utilizada a estatística descritiva. Para verificar diferenças entre os sexos foi aplicado um teste $\mathrm{t}$ de Student para amostras independentes. A diferença das médias de percepções entre as classes socioeconômicas (alta, média e baixa) foi realizada através de Análise de Variância (one-way) e, como post-hoc, o teste de Tukey, em ambos os casos considerando um nível de significância de $p<0,05$. Para testar a associação da insatisfação corporal em relação às classes sociais, foram calculadas as razões de prevalências utilizando o modelo de regressão de Poisson com intervalos de confiança de 95\%, considerando a insatisfação com a imagem corporal como variável desfecho. Na análise da insatisfação corporal com a magreza foi considerada como referência a classe baixa, já para a insatisfação corporal pelo excesso de peso a classe alta foi considerada como referência em função das dife-rentes prevalências encontradas entre as classes sociais. Utilizou-se o teste de Levene para avaliar a homogeneidade entre os grupos considerando as variáveis idade e sexo.

\section{Resultados}

As médias de idade ( \pm DP) dos grupos masculino e feminino foram 11,43 $(2,08)$ e 11,29 $(1,9)$ anos, respectivamente, e apresentaram homogeneidade $(p=0,486)$. As medianas da idade foram: grupo geral (11,48 anos); grupo masculino (11,62 anos) e grupo feminino (11,39 anos). Aproximadamente $41 \%$ $(n=165)$ dos avaliados pertenciam à classe alta, $32 \%$ $(\mathrm{n}=128)$ à classe média e $27 \%(\mathrm{n}=109)$ à classe baixa.

$\mathrm{Na}$ Tabela 1 foram apresentadas as prevalências de sobrepeso e obesidade de acordo com o nível socioeconômico, que indicaram maior prevalência de sobrepeso/obesidade na classe alta, para o sexo masculino $(p=0,002)$.

As médias gerais da PICR foram 3,25 $(1,26)$ para o grupo masculino e $2,87(1,18)$ para o grupo feminino e apresentaram diferenças significativas $(p=0,002)$. Para a PICI, a média para o grupo masculino foi de $3,34(0,97)$ e $2,43(0,8)$ para o grupo feminino; também apresentando diferenças $(p<0,001)$. Na Tabela 2 foram apresentadas as frequências da percepção da imagem corporal por sexo e nível socioeconômico. Nota-se que, para o sexo masculino, nas classes socioeconômicas alta e média, a silhueta 3 da PICR é a mais frequente, enquanto para a classe baixa, a mais frequente é a 4 . No sexo feminino, foi verificado um comportamento diferente: as classes alta e baixa apontaram como PICR a silhueta 2, enquanto a classe média apontou a 3 .

Em relação à percepção da imagem corporal ideal (PICI), pôde-se observar que, independentemente da classe socioeconômica, a silhueta apontada como ideal pelo sexo masculino foi a 3 , enquanto 
para o feminino foi a 2 .

$\mathrm{Na}$ Figura 2 foram apresentados os resultados de distribuição da PICR e PICI de acordo com a idade. No grupo masculino não foram observadas diferenças significativas para as faixas etárias. No grupo feminino o comportamento das curvas foi diferenciado e a média da PICR foi maior que da PICI na maioria das faixas etárias investigadas. As diferenças significativas foram observadas na faixa dos 10 anos $(\mathrm{t}=2,525 ; p=0,016), 11$ anos $(\mathrm{t}=3,415 ; p=0,001)$ e 12 anos $(\mathrm{t}=2,630 ; p=0,012)$.

A análise de variância permitiu identificar diferenças em relação à imagem corporal em algumas situações, de acordo com os resultados apresentados na Tabela 3. O grupo masculino apresentou diferenças estatisticamente significativas apenas na PICR, especificamente entre as classes alta e baixa $(p=0,047)$. O grupo feminino não apresentou diferenças entre as classes socioeconômicas.

Tabela 1

Prevalências de sobrepeso/obesidade de acordo com o nível socioeconômico. Florianópolis, Santa Catarina, 2006.

\begin{tabular}{|c|c|c|c|c|c|c|c|c|}
\hline \multirow{4}{*}{ Classe } & \multicolumn{8}{|c|}{ Prevalências } \\
\hline & \multicolumn{4}{|c|}{ Masculino } & \multicolumn{4}{|c|}{ Feminino } \\
\hline & \multicolumn{2}{|c|}{ Normal } & \multicolumn{2}{|c|}{$\begin{array}{l}\text { Sobrepeso / } \\
\text { Obesidade }\end{array}$} & \multicolumn{2}{|c|}{ Normal } & \multicolumn{2}{|c|}{$\begin{array}{l}\text { Sobrepeso / } \\
\text { Obesidade }\end{array}$} \\
\hline & $\mathrm{n}$ & $\%$ & $\mathrm{n}$ & $\%$ & $\mathrm{n}$ & $\%$ & $\mathrm{n}$ & $\%$ \\
\hline Alta & 34 & 68,0 & 16 & 32,0 & 52 & 86,7 & 8 & 13,3 \\
\hline Média & 55 & 88,7 & 7 & 11,3 & 56 & 84,8 & 10 & 15,2 \\
\hline Baixa & 64 & 90,1 & 7 & 9,9 & 84 & 90,3 & 9 & 9,7 \\
\hline$p^{*}$ & \multicolumn{4}{|c|}{0,002} & \multicolumn{4}{|c|}{0,563} \\
\hline
\end{tabular}

* Teste do qui-quadrado.

Tabela 2

Frequência (\%) de indicação das silhuetas segundo sexo e nível socioeconômico. Florianópolis, Santa Catarina, 2006.

\begin{tabular}{|c|c|c|c|c|c|c|c|c|c|c|}
\hline \multirow{2}{*}{ Percepção da Imagem Corporal } & \multirow{2}{*}{ Classe } & \multicolumn{8}{|c|}{ Silhuetas (\%) } & \multirow[b]{2}{*}{9} \\
\hline & & 1 & 2 & 3 & 4 & 5 & 6 & 7 & 8 & \\
\hline Percepção de Imagem Corporal Real & Alta & 9,3 & 28,0 & 33,4 & 20,0 & 4,0 & 4,0 & 1,3 & 0 & 0 \\
\hline \multirow[t]{2}{*}{ Masculino } & Média & 4,8 & 21,0 & 32,2 & 21,0 & 14,5 & 6,5 & 0 & 0 & 0 \\
\hline & Baixa & 2,2 & 21,7 & 23,9 & 28,3 & 21,7 & 2,2 & 0 & 0 & 0 \\
\hline \multicolumn{11}{|l|}{ Percepção de Imagem Corporal Real } \\
\hline \multirow[t]{3}{*}{ Feminino } & Alta & 5,6 & 42,2 & 36,7 & 10,0 & 2,2 & 2,2 & 1,1 & 0 & 0 \\
\hline & Média & 4,6 & 31,8 & 33,3 & 21,2 & 4,6 & 3,0 & 1,5 & 0 & 0 \\
\hline & Baixa & 4,8 & 47,6 & 12,7 & 28,5 & 4,8 & 0 & 0 & 0 & 1,6 \\
\hline \multicolumn{11}{|l|}{ Percepção de Imagem Corporal ideal } \\
\hline \multirow[t]{3}{*}{ Masculino } & Alta & 5,3 & 10,7 & 44,0 & 29,3 & 10,7 & 0 & 0 & 0 & 0 \\
\hline & Média & 3,2 & 6,4 & 45,2 & 32,3 & 11,3 & 0 & 0 & 1,6 & 0 \\
\hline & Baixa & 0 & 17,4 & 45,7 & 32,6 & 4,3 & 0 & 0 & 0 & 0 \\
\hline Percepção de Imagem Corporal ideal & Alta & 4,4 & 55,6 & 27,8 & 11,1 & 1,1 & 0 & 0 & 0 & 0 \\
\hline \multirow[t]{2}{*}{ Feminino } & Média & 7,6 & 50,0 & 28,8 & 12,1 & 1,5 & 0 & 0 & 0 & 0 \\
\hline & Baixa & 9,5 & 57,1 & 28,6 & 3,2 & 1,6 & 0 & 0 & 0 & 0 \\
\hline
\end{tabular}


Distribuição dos valores de média da PICR e da PICl de acordo com idade para o sexo masculino e feminino.

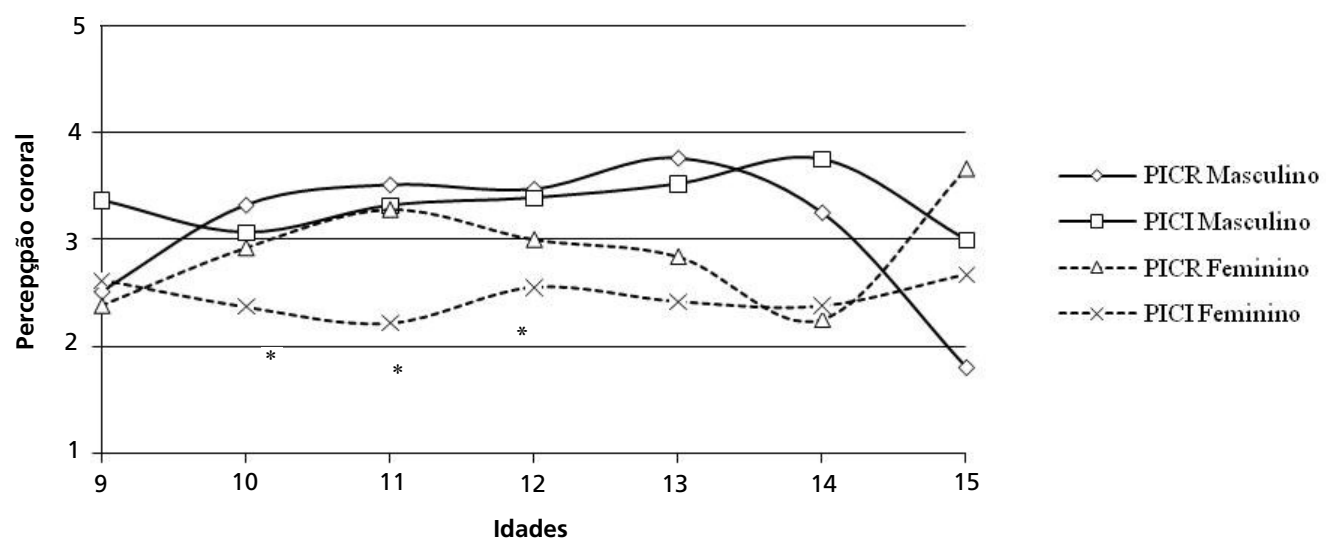

* Diferenças significativas para o sexo feminino; PICR = Percepção de imagem corporal real; PICI = Percepção de imagem corporal ideal.

Tabela 3

Valores médios, desvios padrões e resultados do teste de Tukey para comparação da Percepção de imagem corporal, Percepção da imagem ideal e nível de satisfação entre as classes sociais. Florianópolis, Santa Catarina, 2006.

\begin{tabular}{lcc}
\hline & Masculino & Feminino \\
\cline { 2 - 3 } Variáveis & $\bar{X} \pm \mathrm{DP}$ & $\overline{\mathrm{X}} \pm \mathrm{DP}$ \\
\hline Percepção de imagem corporal real & & \\
Classe alta & $2,99 \pm 1,27 \mathrm{a}$ & $2,72 \pm 1,07 \mathrm{a}$ \\
Classe média & $3,38 \pm 1,28 \mathrm{a}, \mathrm{b}$ & $3,04 \pm 1,19 \mathrm{a}$ \\
Classe baixa & $3,52 \pm 1,18 \mathrm{~b}$ & $2,90 \pm 1,31 \mathrm{a}$ \\
$p^{*}$ & 0,047 & 0,236 \\
Percepção de imagem corporal ideal & & \\
Classe alta & $3,29 \pm 0,98^{\mathrm{a}}$ & $2,48 \pm 0,79 \mathrm{a}$ \\
Classe média & $3,50 \pm 1,06 \mathrm{a}$ & $2,50 \pm 0,86 \mathrm{a}$ \\
Classe baixa & $3,23 \pm 0,79 \mathrm{a}$ & $2,30 \pm 0,75 \mathrm{a}$ \\
$p^{*}$ & 0,313 & 0,281 \\
Nível de satisfação & & $0,23 \pm 1,20 \mathrm{a}$ \\
Classe alta & $-0,30 \pm 1,37 \mathrm{a}$ & $0,54 \pm 1,13 \mathrm{a}$ \\
Classe média & $-0,11 \pm 1,49 \mathrm{a}$ & $0,60 \pm 1,36 \mathrm{a}$ \\
Classe baixa & $0,28 \pm 1,19 \mathrm{a}$ & 0,130 \\
$p^{*}$ & 0,075 & \\
\hline
\end{tabular}

$p^{*}=$ comparações: análise de variância; a,b = diferenças significantes (teste de Tukey). 
Razões de prevalências (RP) e intervalos de confiança (IC95\%) para os grupos masculino, feminino e geral, considerando a insatisfação corporal pela magreza e pelo excesso de peso. Florianópolis, Santa Catarina, 2006.

\begin{tabular}{|c|c|c|c|c|c|c|}
\hline \multirow{2}{*}{ Classe socioeconômica } & \multicolumn{3}{|c|}{ Insatisfação corporal pela magreza } & \multicolumn{3}{|c|}{ Insatisfação corporal pelo excesso de peso } \\
\hline & $\%$ & RP (IC95\%) & $p^{*}$ & $\%$ & RP (IC95\%) & $p^{*}$ \\
\hline \multicolumn{7}{|l|}{ Masculino } \\
\hline Alta & 38,7 & $1,78(0,96-3,30)$ & & 26,7 & 1 & \\
\hline Média & 40,3 & $1,86(0,99-3,47)$ & 0,092 & 37,1 & $1,39(0,85-2,29)$ & 0,145 \\
\hline Baixa & 21,7 & 1 & & 43,5 & $1,63(0,98-2.69)$ & \\
\hline \multicolumn{7}{|l|}{ Feminino } \\
\hline Alta & 26,7 & $2,81(1,21-6,46)$ & & 38,9 & 1 & \\
\hline Média & 13,6 & $1,43(0,54-3,80)$ & 0,014 & 48,5 & $1,24(0,86-1,76)$ & 0,314 \\
\hline Baixa & 9,5 & 1 & & 50,8 & $1,29(0,90-1,84)$ & \\
\hline \multicolumn{7}{|l|}{ Geral } \\
\hline Alta & 32,1 & $2,18(1,32-3.62)$ & & 33,3 & 1 & \\
\hline Média & 26,6 & $1,81(1,06-3,10)$ & 0,005 & 43,0 & $1,29(0,95-1,71)$ & 0,050 \\
\hline Baixa & 14,7 & 1 & & 47,7 & $1,43(1,06-1,90)$ & \\
\hline
\end{tabular}

* Valor de $p$ não ajustado (qui-quadrado).

Na Tabela 4 foram apresentados os resultados das prevalências e razões de prevalências referentes à insatisfação com a imagem corporal entre as classes socioeconômicas. Os resultados foram analisados separadamente por sexo e considerando o grupo geral. Na classe baixa, no grupo masculino, existiu maior insatisfação pelo excesso de peso, enquanto nas classes alta e média a maior insatisfação foi pela magreza. No grupo feminino, a maior insatisfação foi em relação ao excesso de peso nas três classes avaliadas. Cabe ressaltar que a classe baixa, para ambos os sexos, foi a que apresentou menor insatisfação pela magreza. Além disso, o grupo feminino apresentou percentuais maiores de satisfação corporal nas três classes sociais que o masculino.

Em relação à insatisfação corporal pela magreza o grupo feminino apresentou diferenças entre as classes baixa e alta $(p=0,014)$ tendo a classe alta apresentado prevalência 2,81 vezes maior em relação à classe baixa. No grupo geral, foram identificadas associações significativas entre todas as classes, sendo que, as classes alta e média, apresentaram maior insatisfação que a classe baixa. Observou-se que, em relação à insatisfação corporal pelo excesso de peso, houve uma tendência de maior insatisfação na classe baixa, considerando o grupo geral $(p=0,050)$.

\section{Discussão}

As principais limitações apontadas nesse estudo referem-se ao método de avaliação da imagem corporal e ao não controle do estágio maturacional da amostra. A avaliação da percepção da imagem corporal pelo método de silhuetas é um modelo clássico de pesquisa e apresenta diferentes possibilidades de análise. Porém, o método apresenta a impossibilidade de adaptação das figuras às diferentes faixas etárias e a limitação de identificação da imagem por se tratar de um parâmetro bidimensional em preto e branco. Acredita-se que a utilização de figuras adaptadas às diferentes faixas etárias e com apresentações tridimensionais poderia, em tese, melhorar a medida da real percepção corporal. Por outro lado o controle do estágio maturacional poderia fortalecer os resultados estatísticos, já que os adolescentes parecem apresentar maior insatisfação com a sua imagem corporal.

Considerando os valores médios do grupo, foi verificada uma tendência dos meninos idealizarem silhuetas maiores e as meninas silhuetas menores, confirmando os dados apresentados pela literatura. 19,20 Esse comportamento também foi identificado por outros estudos que discutem a influência social e da mídia, que levam os meninos a desejarem uma silhueta maior, indicando corpos mais fortes, e 
as meninas corpos menores, ou seja, mais magros. ${ }^{21-}$ 23 Apesar disso, os percentuais de insatisfação desse estudo foram maiores que os encontrados em escolares de 7 a 19 anos de alguns municípios do interior do Estado de Minas Gerais, cuja insatisfação foi identificada em 59\% dos jovens. 19

Em relação às classes socioeconômicas identificou-se que, proporcionalmente ao número de indivíduos em cada classe, os meninos da classe baixa se perceberam (PICR) com uma silhueta maior em relação à classe alta mesmo que as prevalências de sobrepeso e obesidade dos grupos indiquem o contrário. Nas meninas esta diferença não foi verificada. Um comportamento diferenciado entre indivíduos do sexo masculino e feminino também foi identificado por Wardle et al.24 e Wolfe e Smith, 25 que verificaram que os meninos têm apresentado menos esforços no controle do peso corporal em relação às meninas. Apesar disso, em relação à PICI, não houve diferenças considerando nenhuma classe ou grupo, ou seja, os jovens, independente da classe social, almejam silhuetas similares.

Embora uma análise aprofundada sobre o real estado nutricional não tenha sido objetivo do presente estudo, os dados de prevalência de sobrepeso /obesidade da amostra identificados no município de Florianópolis foram altos e confirmaram os resultados de levantamentos anteriores, 26 o que remete à necessidade de intervenções voltadas à saúde dos escolares. Nesta amostra, as maiores prevalências de obesidade foram identificadas na classe social alta, principalmente nos meninos.

Considerando a idade, foi possível verificar a tendência de as meninas desejarem corpos mais magros e os meninos corpos mais fortes. Essa diferença entre os sexos pode estar relacionada a um fator cultural, no qual os rapazes são estimulados a desenvolverem outras competências sociais, enquanto as moças são instigadas a praticarem atividades que impliquem perda de peso com enfoque no caráter estético. ${ }^{2}$ No grupo masculino, na idade de 12 anos foi identificada a maior semelhança entre a PICR e a PICI, enquanto no grupo feminino as percepções reais e ideais foram mais semelhantes aos 14 anos. No entanto, é preciso destacar que nas idades de 14 e 15 anos o baixo número de sujeitos pode interferir nos resultados. Foi possível observar também, que na faixa etária dos nove anos, independente do sexo, os indivíduos identificavam como ideais silhuetas maiores às suas, o que pode estar relacionado ao fato de serem crianças e não terem iniciado o processo de maturação sexual que causa grandes transformações e diferenciações corporais. 27
Em relação à insatisfação com a imagem corporal, considerando as análises de variância e de prevalência de insatisfação, os dados precisam ser analisados com critério. Percentualmente a maior parte das meninas da classe baixa se apresentou insatisfeita pelo excesso de peso. Esses resultados são contrários aos apresentados por Jackson et al., ${ }^{28}$ que investigando 340 moças escolares verificaram maior incidência de sobrepeso e obesidade, bem como, desejo por um corpo mais magro nas classes sociais mais altas. Um dado importante apontado pela literatura relacionado a esse tema é que nem todas as meninas que estão insatisfeitas com a gordura estão com o peso fora do normal, e esse olhar de sua imagem corporal implica uma distorção cognitiva que pode ser explicada por algum tipo de aprendizado imposto a um grupo por pressão psicossocial e cultural. 29

Os resultados encontrados nos escolares de Florianópolis se mostram convergentes com investigações internacionais, como, por exemplo, o estudo de Wang et al.,7 que em um grupo de 768 jovens de 10 a 18 anos (chineses, vietnamitas, italianos e gregos), observaram que as crianças e adolescentes com menor renda tenderam a desejar corpos mais magros. No entanto, é importante destacar que, embora exista uma tendência de maior insatisfação paras as classes mais baixas, não há uma coerência com esta insatisfação com as prevalências de sobrepeso/obesidade, especialmente no grupo masculino. Os meninos da classe alta apresentam maior prevalência de sobrepeso/obesidade e uma maior insatisfação com a magreza. Esse resultado pode estar ligado às próprias silhuetas do protocolo e ao padrão de beleza masculino, que exige cada vez mais corpos fortes, e até mesmo as dificuldades dos meninos nessa faixa etária de perceberem suas próprias dimensões corporais. Já no grupo feminino, a relação insatisfação com o excesso de peso e prevalência de sobrepeso/obesidade apresentou maior coerência.

Os resultados encontrados em Florianópolis convergem em parte com os dados de O'Dea, 12 em um estudo com crianças e adolescentes de escolas públicas e privadas na Austrália, no qual o autor identificou maiores percentuais de crianças insatisfeitas com a imagem corporal nas classes sociais menos favorecidas mas, no entanto, as prevalências de sobrepeso/obesidade também foram maiores nessa classe. O'Dea12 destaca que a insatisfação com a imagem corporal teve uma forte influência cultural, ou seja, as crianças de territórios e grupos étni$\cos$ em que o sobrepeso e a obesidade são mais 
comuns e aceitos se apresentaram menos insatisfeitas, e um menor percentual, principalmente de meninas, se consideraram com excesso de peso, mesmo com estado nutricional semelhante. Comparando as realidades sociais à brasileira e à australiana, pode-se inferir que o Brasil está passando por um momento de transição nutricional, justificando os resultados obtidos em Florianópolis.

Da mesma forma, resultados de investigações com crianças e adolescentes de países europeus em sua maioria convergem para um aumento, tanto das prevalências de sobrepeso/obesidade, como da insatisfação com a imagem corporal nas classes menos favorecidas. Sujoldzić e De Lucia, ${ }^{4}$ em uma análise da insatisfação com a imagem corporal em diversos países europeus, identificaram relações significativas e maiores prevalências de valores altos de IMC, hábitos alimentares menos saudáveis e insatisfação com a imagem corporal nos jovens com nível socioeconômico mais baixo. No entanto, como grande parte dos estudos, não se analisou insatisfação pela magreza e pelo excesso de peso em separado.

Hölling e Schlack, 10 em estudo realizado na Alemanha, identificaram que $21,9 \%$ das crianças e adolescentes de 11 a 17 anos apresentaram sintomas de transtornos alimentares, sendo que os jovens com baixo nível socioeconômico são $27,6 \%$ mais afetados por esses distúrbios. Bauer e Kirchengast, 8 investigando jovens austríacos, identificaram prevalências de sobrepeso e obesidade significativamente menores em crianças e adolescentes com alto nível socioeconômico e educacional da família. Da mesma forma Paxton et al.,5 na Alemanha, identificaram uma tendência dos jovens com baixo nível socioe-

\section{Referências}

1. Cash TF, Pruzinski T. Body images $\neg$ development, deviance, and change. New York: Guiford; 1990.

2. Ricciardelli LA, Mccabe MP, Banfield S. Sociocultural influences on body image and body changes methods. J Adolesc Health. 2000; 26: 3-4.

3. Conti MA, Frutuoso MFP, Gambardella AMD. Excesso de peso e insatisfação corporal em adolescentes. Rev Nutr. 2005; 18: 491-7.

4. Sujoldzić A, De Lucia A. A cross-cultural study of adolescents-BMI, body image and psychological well-being. Coll Antropol. 2007; 31: 123-30.

5. Paxton SJ, Eisenberg ME, Neumark-Sztainer D. Prospective predictors of body dissatisfaction in adolescent girls and boys: a five-year longitudinal study. Dev Psychol. 2006; 42: 888-99. conômico terem maior insatisfação com a imagem corporal. Apesar desses resultados, Wang et al.,7 estudando crianças e adolescentes orientais, não observaram diferenças significativas nos indicadores de insatisfação com o corpo conforme o nível socioeconômico, o que indica particularidades dessas relações em diferentes realidades.

A literatura ainda é restrita no sentido de explicar as reais influências do nível socioeconômico nas questões biológicas. A influência do nível socioeconômico, no controle de peso e na satisfação /descontentamento com a imagem corporal, segundo Kumanyika, ${ }^{30}$ pode ocorrer por inúmeros fatores, como a possibilidade de comprar alimentos saudáveis, segurança e oportunidades para a prática da atividade física e de esportes, bem com a possibilidade de contato com profissionais especializados para acompanhamento. Apesar disso, mais resultados, em diferentes culturas, são necessários para uma análise mais aprofundada do tema.

De forma geral foi observada uma tendência dos meninos desejarem corpos mais fortes e das meninas corpos mais magros. Os escolares das classes mais altas apresentaram maiores prevalências de insatisfação com a magreza, e a insatisfação com o excesso de peso foi mais marcante nas classes mais baixas. As análises remetem a comportamentos diferenciados da percepção da imagem corporal dos jovens, considerando insatisfação pelo excesso de peso e pela magreza, o sexo, o real estado nutricional e o nível socioeconômico, existindo, dessa forma, a necessidade de avaliação desses parâmetros em futuras pesquisas com percepção da imagem corporal em crianças e adolescentes.
6. Robinson TN, Chang JY, Haydel KF, Killen JD. Overweight concerns and body dissatisfaction among thirdgrade children: the impacts of ethnicity and socioeconomic status. J Pediatr. 2001; 138: 181-7.

7. Wang Z, Byrne NM, Kenardy JA, Hills AP. Influences of ethnicity and socioeconomic status on the body dissatisfaction and eating behaviour of Australian children and adolescents. Eat Behav. 2005; 6: 23-33.

8. Bauer M, Kirchengast S. Body composition, weight status, body image and weight control practices among female adolescents from eastern Austria. Anthropol Anz. 2006; 64: 321-31.

9. Erickson SJ, Gerstle M. Investigation of ethnic differences in body image between Hispanic/biethnic-Hispanic and non-Hispanic White preadolescent girls. Body Image. 2007; 4: 69-78. 
10. Hölling H, Schlack R. Eating disorders in children and adolescents. First results of the German Health Interview and Examination Survey for Children and Adolescents (KiGGS). Bundesgesundheitsblatt Gesundheitsforschung Gesundheitsschutz. 2007; 50: 794-9.

11. Triches RM, Giugliani ERJ. Insatisfação corporal em escolares de dois municípios da região Sul do Brasil. Rev Nutr. 2007; 20: 119-28

12. O'Dea JA. Gender, ethnicity, culture and social class influences on childhood obesity among Australian schoolchildren: implications for treatment, prevention and community education. Health Soc Care Community. 2008; 16: 282-90.

13. IBGE (Instituto Brasileiro de Geografia e Estatísticas). Pesquisa de orçamentos familiares no Brasil, 2002/2003. Antropometria e análise do estado nutricional de crianças e adolescentes no Brasil. Rio de Janeiro; 2006.

14. PNUD (Programa das Nações Unidas para o Desenvolvimento), IPEA (Instituto de Pesquisa Econômica Aplicada), IBGE (Instituto Brasileiro de Geografia e Estatística), Fundação João Pinheiro. Atlas do desenvolvimento humano no Brasil: índice de desenvolvimento humano municipal. Brasília, DF; 2003.

15. Luiz RR, Magnanini, MMF. A lógica da determinação do tamanho da amostra em investigações epidemiológicas. Cad Saúde Coletiva. 2000; 8: 9-28.

16. Stunkard AJ, Sorenson T, Schlusinger F. Use of the Danish adoption register for the study of obesity and thinness. In: Kety SS, Rowland LP, Sidman RL, Mat-thysse SW, editors. The genetics of neurological and psychiatric disorders. New York: Raven; 1983. p. 115-20.

17. ANEP (Associação Nacional de Empresas de Pesquisa). Critérios de classificação econômica no Brasil. 2003 [acesso em 10 mar. 2007]. Disponível em: http://www.abep. org.br

18. Cole TJ, Bellizzi MC, Flegal KM, Dietz WH. Establishing a standard definition for child overweight and obesity worldwide: international survey. BMJ. 2000; 320: 1240-3.

19. Vilela JEM, Lamounier JA, Dellaretti Filho MA, Barros Neto JR, Horta GM. Transtornos alimentares em escolares. J Pediatr. (Rio J). 2004; 80: 49-54.

Recebido em 19 de março de 2008

Versão final apresentada em 10 de junho de 2009

Aprovado em 19 de junho de 2009
20. Pinheiro AP, Guigliano ERJ. Quem são as crianças que se sentem gordas apesar de terem peso adequado? J Pediatr. (Rio J). 2006; 82: 232-5.

21. Ogden J, Evans C. The problem with weighing: effects on mood, self-esteem and body image. Int J Obes Relat Metab Disord. 1996; 20: 272-7.

22. Foster GD, Wadden TA, Vogt RA. Body image in obese before, during, and after weight loss treatment. Health Psychol. 1997; 16: 226-9.

23Leonhard ML, Barry NJ. Body image and obesity: effects of gender and weight on perceptual measures of body image. Addict Behav. 1998; 23: 31-4.

24. Wardle J, Haase A, Steptoe A, Nillapun M, Jonwutiwes K, Bellisle F. Gender differences in food choices: the contribution of health beliefs and dieting. Ann Behav Med. 2004; 27: 107-16.

25. Wolfe BL, Smith JE. Different strokes for different folks: why overweight men do not seek weight loss treatment. Eat Disord. 2002; 10: 115-24.

26. Soar C, Vasconcelos FAG, Assis MAA, Grosseman S, Luna MEP. Prevalência de sobrepeso e obesidade em escolares de uma escola pública de Florianópolis, Santa Catarina. Rev Bras Saúde Matern Infant. 2004; 4: 391-7.

27. Malina RM, Bouchard C, Bar-Or O. Growth, maturation, and physical activity. 2. ed. Champaign: Human Kinetics; 2004.

28. Jackson RT, Rashed M, Saad-Eldin R. Rural urban differences in weight, body image, and dieting behavior among adolescent Egyptian schoolgirls. Int J Food Sci Nutr. 2003; 54: $1-11$.

29. Nunes MA, Olinto MTA, Barrosa FC, Camey S. Influência da percepção do peso e do índice de massa corporal nos comportamentos alimentares anormais. Rev Bras Psiquiatr. 2001; 23: 21-7.

30. Kumanyika SK. Obesity treatment in minorities. In: Wadden TA, Stunkard AJ, editors. Handbook of obesity treatment. New York: Guilford; 2002. p. 416-46. 